\title{
Effectiveness of the bevacizumab-irinotecan regimen in the treatment of recurrent glioblastoma multiforme: Comparison with other second-line treatments without this regimen
}

\author{
DANIEL RUIZ-SÁNCHEZ ${ }^{1}$, MIGUEL ALAGUERO CALERO ${ }^{1}$, ALEJANDRO JOSÉ SASTRE-HERES ${ }^{1}$, \\ MARÍA TERESA IGLESIAS GARCÍA ${ }^{1}$, MIGUEL ANGEL CALLEJA HERNANDEZ ${ }^{2}$, \\ FERNANDO MARTÍNEZ MARTINEZ ${ }^{3}$ and JAIME PEÑA-DÍAZ ${ }^{3}$ \\ ${ }^{1}$ Hospital Pharmacy Department, Central University Hospital of Asturias, Oviedo, Asturias 33006;
${ }^{2}$ Hospital Pharmacy Department, Virgen de las Nieves Hospital, Granada 18014;
${ }^{3}$ Faculty of Pharmacy, University of Granada, Granada 18071, Spain
}

Received May 2, 2012; Accepted August 2, 2012

DOI: $10.3892 / \mathrm{ol} .2012 .861$

\begin{abstract}
A retrospective cohort study was conducted to analyse the effectiveness of bevacizumab and irinotecan (BVZ/ CPT-11) as a second-line treatment in patients with primary glioblastoma multiforme (GBM) in comparison with a control group that were not administered BVZ/CPT-11 at the first recurrence. The difference in overall survival (OS) between the two groups was used as a predictor of effectiveness. OS was calculated according to prognostic factors and gender. A total of 28 and 32 patients were enrolled in the BVZ/CPT-11 cohort and control group, respectively. The median OS was 17.94 months (95\% CI, 14.91-20.96) in the BVZ/CPT-11 treatment cohort and 10.97 months (95\% CI, 7.65-14.30) in the control cohort. The results obtained on the effectiveness of BVZ/CPT-11 treatment in patients with primary GBM are consistent with data from previous studies. No significant differences were identified in OS based on prognostic factors; therefore, the latter cannot be used to select patients who would incur the greatest benefits from BVZ/CPT-11 treatment.
\end{abstract}

\section{Introduction}

Glioblastoma multiforme (GBM) is a type of cancer that affects the glial cells in the central nervous system (CNS). It is the most common (1) and aggressive type of primary brain tumour in adults and its outcome is fatal (2).

In 2010, there were 22,020 new cases of primary brain tumours diagnosed in the USA and approximately 13,140 mortalities (3). The incidence of brain tumours has increased

Correspondence to: Mr. Daniel Ruiz-Sánchez, Hospital Pharmacy Department, Hospital Universitario Central de Asturias, Julián Clavería, Oviedo, Asturias 33006, Spain

E-mail: danrusa1985@gmail.com

Key words: glioblastoma, bevacizumab, irinotecan, effectiveness over the past 30 years, particularly in the elderly (2). GBM is the most common type of brain tumour in adults and accounts for 54\% of all gliomas (1). The highest incidence is identified in adults over the age of 45 years (5). The prevalence is higher in males compared with females, and in two studies conducted in France (6) and the USA (7), the male to female ratio was 1.6:1 and 1.48:1, respectively.

The Hospital Universitario Central de Asturias (HUCA) is the referral hospital in Asturias (Spain). Among the 1 million inhabitants of Asturias, 4,580 tumours were diagnosed in 2008 , of which $3 \%$ were brain tumours. GBM had the highest incidence and accounted for $19.3 \%$ of all brain tumours (8).

More than $90 \%$ of GBMs are primary tumours. They generally have a clinical history of less than one year and are more frequent in elderly patients. Despite a multitude of scientific advances, the median survival time for these patients receiving standard treatment [surgical resection, radiotherapy (RT) and temozolomide (TMZ), followed by six maintenance cycles of TMZ] remains low, at approximately 14 months $(9,10)$.

The prognosis for patients with GBM appears to be influenced by a number of factors. Certain factors that have been suggested to predict a good prognosis include age (adults under 58 years have better rates of survival and progression-free disease) (11), Karnofsky Performance Status (KPS) $\geq 70-80 \%$ at diagnosis (12), absence of anaplastic cells, existence of oligodendroglial elements, tumour size and type of surgical resection (complete resections have been identified to have better survival data) (13), and preserved cognitive status (10).

The use of bevacizumab and irinotecan (BVZ/CPT-11) in GBM is based on the fact that these tumours are highly vascularised, and preclinical data demonstrate that glioma growth is dependent on tumour-associated neovascularisation $(14,15)$. In the 1970s, Folkman (16) proposed the hypothesis of using angiogenesis as an anticancer therapeutic target. Based on these theoretical premises, it appears reasonable to use an antivascular endothelial growth factor agent (anti-VEGF), including BVZ, for this type of tumour. However, anti-VEGF is rarely used in GBM. BVZ studies are now at phase II and it appears that in addition to being active in GBM, it is also well 
tolerated $(17,18)$. The antiglioma biological activity of BVZ has also been demonstrated (19), with results superior to those obtained in previous studies (20-35).

In May 2009, the US Food and Drug Administration (FDA) approved the use of BVZ as a single agent for patients diagnosed with GBM and disease progression who were previously treated with the standard first-line treatment (36). However, BVZ is not yet administered for any line of GBM treatment in Europe, with results pending from phase III studies to confirm efficacy data.

\section{Materials and methods}

Study design. A retrospective cohort study with a control group was conducted to compare the effectiveness of two chemotherapy treatments in terms of overall survival (OS) in two patient cohorts. The study was approved by the ethics committee of Central University Hospital of Asturias, Asturias, Spain. Consent was obtained for use of patient data.

\section{Study population}

Patients. Patients diagnosed with and treated for primary GBM between 2002 and 2009 at HUCA were selected for this study. The control cohort included all patients treated with TMZ between January 2002 and December 2006 at the Hospital Pharmacy Service (HUCA), and who met the inclusion criteria. The BVZ/CPT-11 cohort included all patients diagnosed with any type of glioma and treated with the study regimen as second-line treatment between January 2007 and December 2009, and who met the inclusion criteria.

Inclusion criteria. All patients were histologically diagnosed with primary GBM between 2002 and 2009 at HUCA. Patients were over the age of 18 and underwent surgical procedures regardless of the type of resection, including biopsies. The control cohort consisted of patients who received a first-line treatment with TMZ concomitantly with RT and/or maintenance cycles that were conducted until completion or progression. The control patients did not receive second-line treatment or the second-line treatment differed from that of the study cohort. The study cohort consisted of patients who met the control cohort first-line inclusion criteria and also received BVZ/CPT-11 regimen as second-line treatment.

Exclusion criteria. Patients who did not meet all the inclusion criteria and those lacking any data required for analysis were excluded from the study.

\section{Definition of endpoints}

Primary endpoints (quantitative variables). The primary efficacy endpoint of the study was OS, which was calculated from the time of diagnosis to the date of the last observation in the patient's medical record or mortality. Interim survival (IS) following second-line treatment with BVZ/CPT-11 was measured from the start of the second-line treatment to the date of the last observation or mortality. Time to progression (TTP) following first-line treatment was also calculated, representing the time from the start of treatment until the time of disease progression as defined by the oncologist.

Secondary endpoints (dichotomous and qualitative variable). The secondary endpoint was the type of second-line treatment received, identifying whether the treatment included the BVZ/CPT-11 regimen.
Table I. Patient clinical and demographic characteristics.

\begin{tabular}{lccc}
\hline Variable & $\begin{array}{c}\text { With BVZ } \\
(\%)\end{array}$ & $\begin{array}{c}\text { Without BVZ } \\
(\%)\end{array}$ & P-value \\
\hline $\begin{array}{l}\text { No. of patients } \\
\text { Age (years) }\end{array}$ & $46.7(28)$ & $53.3(32)$ & \\
Median & 52.6 & 56.1 & 0.246 \\
Range & $23-69$ & $27-73$ & \\
Gender & & & 0.741 \\
Male & $60.7(17)$ & $53.1(17)$ & \\
Female & $39.3(11)$ & $46.9(15)$ & \\
KPS (\%) & & & \\
Median & 70 & 70.729 \\
Range & $60-90$ & $60-100$ & \\
Type of resection & & & \\
Complete & $46.4(13)$ & $43.7(14)$ & \\
Partial & $42.9(12)$ & $43.7(14)$ & \\
Biopsy & $10.7(2)$ & $9.3(3)$ & \\
ND & & $3.1(1)$ & \\
\hline
\end{tabular}

BVZ, bevacizumab; KPS, Karnofsky performance status; ND, no data.

Data analysis. An assessment of differences in effectiveness was conducted by comparing the OS and TTP values between the cohorts using SPSS version 18.0 (SPSS, Chicago, IL, USA). Firstly, the two study samples were identified, the means and standard deviations were calculated for the quantitative variables and absolute and relative frequencies were calculated for the qualitative variables. OS was identified using the Kaplan-Meier method, which was conducted in each study group, and the curve was plotted using a primary independent variable.

Subsequently, the difference in the survival rate of each group was compared, taking into account each possible risk factor, using the log rank hypothesis. $\mathrm{P}<0.05$ was considered to indicate a statistically significant difference.

\section{Results}

Patients and treatment. The control cohort (without BVZ) consisted of 32 patients. Among the 128 patients initially receiving $\mathrm{TMZ}$, the following were excluded: 25 patients whose diagnosis was not brain tumour, 55 with a diagnosis of low grade glioma, 11 with secondary glioblastoma and six who were diagnosed prior to 2002 .

The study cohort (with BVZ) consisted of 28 patients. Among the 36 patients who were initially diagnosed with glioma and treated with BVZ/CPT-11, 8 patients were excluded when their initial diagnosis did not correspond with primary GBM, which was the subject of study.

The clinical and demographic characteristics of the patients included in the two cohorts are summarised in Table I, and the different treatments that were administered are identified in Table II. 
Table II. First- and second-line treatment received by each cohort.

\begin{tabular}{lcc} 
Treatment (concentration) & With BVZ Without BVZ \\
\hline First-line & 26 & 14 \\
RT + TMZ $\left(100 \mathrm{mg} / \mathrm{m}^{2}\right)+$ & & 11 \\
TMZ $\left(240 \mathrm{mg} / \mathrm{m}^{2}\right) \times 6$ cycles & & 7 \\
RT + TMZ $\left(240 \mathrm{mg} / \mathrm{m}^{2}\right)$ x 6 cycles & 2 & \\
RT+TMZ $\left(100 \mathrm{mg} / \mathrm{m}^{2}\right)$ & - & - \\
Second-line & & - \\
BVZ $(7.5 \mathrm{mg} / \mathrm{kg})+$ & 5 & - \\
CPT-11 $\left(240 \mathrm{mg} / \mathrm{m}^{2}\right) \times 21$ days & & \\
BVZ $(10 \mathrm{mg} / \mathrm{kg})+$ & 9 & 5 \\
CPT-11 $\left(140 \mathrm{mg} / \mathrm{m}^{2}\right) \times 14$ days & 14 & 2 \\
BVZ $(5 \mathrm{mg} / \mathrm{kg})+$ & & 1 \\
CPT-11 $\left(140 \mathrm{mg} / \mathrm{m}^{2}\right)$ x 14 days & - & 24 \\
CPT-11 & - & - \\
CT endotecarin vs. BCNU & - & \\
CT lomustine vs. enzastaurin & - & \\
No further treatment & & \\
\hline BVZ, bevacizumab; RT, radiotherapy; TMZ, temozolomide; CPT-11, \\
irinotecan; CT, clinical trial; BCNU, bischloroethylnitrosourea. \\
\hline
\end{tabular}

Survival and progression. With regard to the primary endpoint, the median OS was 17.94 months [95\% confidence interval (CI), 14.91-20.96] in the BVZ/CPT-11 treatment cohort and 10.97 months (95\% CI, 7.65-14.30) in the control cohort. A comparison of OS in the two groups was plotted on a Kaplan-Meier curve (Fig. 1; $\mathrm{P}=0.001$ ). The IS for patients receiving BVZ/CPT-11 was 8.8 months (95\% CI, 5.3-12.3).

TTP in first-line treatment for the control cohort was 4.07 months (95\% CI, 1.66-6.49) and 7.26 months (95\% CI, 5.51-9.01) in the BVZ cohort ( $\mathrm{P}=0.018$; Fig. 2). The difference in TTP was also calculated following BVZ/CPT-11 administration as second-line treatment. This was identified to be 5.75 months (95\% CI, 4.58-6.92).

The duration of first-line treatment was also compared between the two cohorts. The median duration of TMZ treatment was 6.67 months (95\% CI, 6-287-7.052) in the study cohort and 4.14 months $(95 \% \mathrm{CI}, 1.686-6.594)$ in the control cohort $(\mathrm{P}=0.004)$.

Survival and risk factors. Survival was analysed for these variables by applying possible confounding variables. Table III reveals the median survival with CIs for the two cohorts by gender and age divided into two groups ( $<58$ and $\geq 58$ years). KPS score was also divided into two groups ( $<80$ and $\geq 80 \%)$. Finally, the type of surgical resection was analysed.

\section{Discussion}

As shown in Table I, there are no differences between patients in the two cohorts with regard to variables that may act as confounders. The variables were similar to those identified in other studies. For example, the median age, KPS score,

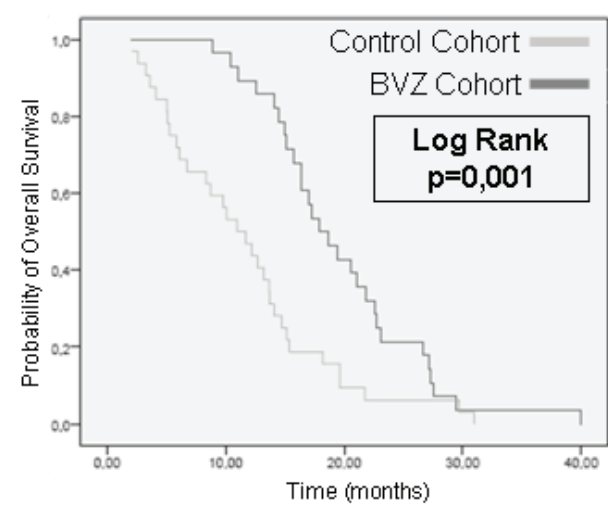

Figure 1. Kaplan-Meier curve revealing the OS in the two cohorts. BVZ, bevacizumab; OS, overall survival.

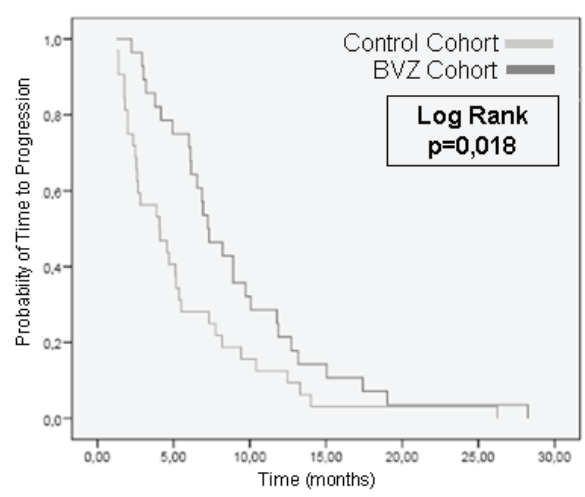

Figure 2. Kaplan-Meier curve revealing the TTP following first-line treatment in the two cohorts. BVZ, bevacizumab; TTP, time to progression.

gender ratio and the different types of resection observed in the two cohorts, were similar to those identified in previous studies $(9,10,17-35)$.

When comparing OS data in patients treated with and without BVZ, it is evident that the median OS from diagnosis increased by almost 7 months in the BVZ treatment cohort $(\mathrm{P}=0.001)$. In relative terms, this represents a 50-60\% increase in OS. However, it should be noted that patients did not receive the same number of first-line treatment cycles in the two cohorts, as discussed below.

Results for OS in the BVZ cohort were similar to those reported in the few phase II trials that were available at the time of writing. Vredenburgh et al (20) and Friedman et al (17) identified that the median OS from the start of BVZ/CPT-11 treatment was 9.8 and 9.2 months, respectively, compared with the 8.8 months demonstrated in our study.

Certain factors may have influenced these small differences. In our study, $17.9 \%$ of patients in the BVZ cohort had a KPS score of $<70 \%$, while other trials established a KPS score of $\geq 70 \%$ as an inclusion criterion (19), and the percentage of these patients was much lower than in our study (20). There were also other criteria, such as $<1.5 \mathrm{mg} / \mathrm{ml}$ bilirubin and a certain platelet count, which we did not take into account. It should also be noted that we did not have the date of mortality for 12 patients, which also may have influenced our lower OS figures.

The OS value of the non-BVZ cohort (10.97 months) was significantly different from the 14 and 15 month OS that was 
Table III. OS data by subgroup for each variable.

\begin{tabular}{|c|c|c|c|c|}
\hline \multirow[b]{2}{*}{ Variable } & \multicolumn{2}{|c|}{ OS $(95 \% \mathrm{CI})$} & \multicolumn{2}{|c|}{ Log-rank (Mantel-Cox) } \\
\hline & Control & BVZ & BVZ & BVZ vs. control \\
\hline \multicolumn{5}{|l|}{ Age (years) } \\
\hline$\geq 58$ & $6.70(4.39-9.02)$ & $18.69(16.49-20.90)$ & 0.602 & 0.022 \\
\hline$<58$ & $14.06(13.29-14.84)$ & $17.25(12.95-21.55)$ & & \\
\hline \multicolumn{5}{|l|}{ Gender } \\
\hline Male & $9.79(5.60-13.98)$ & $18.69(14.63-22.76)$ & 0.627 & 0.100 \\
\hline Female & $14.06(1.38-26.75)$ & $17.25(11.43-23.06)$ & & \\
\hline \multicolumn{5}{|l|}{ KPS (\%) } \\
\hline$\geq 80$ & $13.70(2.50-24.90)$ & $22.57(20.46-24.68)$ & 0.694 & 0.064 \\
\hline$<80$ & $10.97(9.08-12.87)$ & $17.25(15.13-19.37)$ & & \\
\hline \multicolumn{5}{|c|}{ Type of resection } \\
\hline Complete & $14.06(13.48-14.64)$ & $17.94(12.38-23.49)$ & N/D & N/D \\
\hline Incomplete & 9.79 (4.19-15.39) & $16.36(11.23-21.49)$ & & \\
\hline Biopsy & 6.70 & $22.57(6.48-38.66)$ & & \\
\hline
\end{tabular}

An analysis was conducted to identify any differences in survival between the possible risk factors by comparison with the log rank hypothesis. OS, Overall survival; CI, confidence interval; BVZ, bevacizumab; KPS, Karnofsky performance status; N/D, no data.

demonstrated by Stupp et al (9) and Seiz et al (36), respectively. This discrepancy may be attributed to the fact that $66.7 \%$ of the patients in our study were diagnosed and treated before 2005, which is when the study by Stupp et al (9) was published, establishing the grounds for concomitant TMZ and RT and the six maintenance cycles. Until then, fewer cycles of TMZ were administered, usually entailing four 28-day cycles with one to five days of treatment. Additionally, patients who underwent complete resection did not receive cycles concomitantly with RT.

The characteristics of our patients also differed from the study by Stupp et al (9). The latter required adequate renal and liver function tests and blood values within a certain range as an inclusion criterion, and we did not take these parameters into consideration when enrolling patients in our study.

TTP data identified following first-line treatment with TMZ and RT in the BVZ cohort was similar to data from previous studies, which refers to progression-free survival (PFS) of 6.9 months (9). In the non-BVZ cohort, TTP was 4.07 months. The difference between these figures can be explained as previously stated.

It should be noted that TTP is not exactly comparable with PFS. TTP does not take into account mortalities without progression or mortalities from other causes, which are included in PFS. Furthermore, we collected data from the time that the oncologist reported clinical progression, and not when progression was observed on nuclear magnetic resonance (NMR). Therefore, there may be some time bias between the time when the NMR was conducted and the time when the doctor in charge of follow-up confirmed that the image represented disease progression.

By comparing TTP data from the two cohorts, it is apparent that there is a statistically significant difference between the two $(\mathrm{P}=0.018)$. To discover the reason for this difference, we measured the duration of TMZ treatment in both cohorts and, as predicted, the median duration of TMZ treatment in the BVZ cohort was longer than in the control cohort $(\mathrm{P}=0.004)$.

A multivariate analysis did not reveal any statistically significant differences in OS among the variables we considered as confounders. However, the clinically significant differences we identified are noteworthy.

With regard to gender, no differences were identified in either of the cohorts. In terms of age, OS data for the BVZ cohort was almost equal in the two age groups $(<58$ and $\geq 58$ years); however, there were differences in the OS data for the non-BVZ cohort for both age groups. The $<58$-year-old group had an OS of 14 months (95\% CI, 13.27-14.84), while the $\geq 58$-year-old group had an OS of six months (95\% CI, 4.69-7.16). We identified similar data in a previous study (8). Younger patients (under the age of 58) have been reported to have a better prognosis, with better rates of survival and progression-free disease (11). Therefore, it cannot be said that BVZ is more effective in one age group than another, because the data are similar for this cohort.

With regard to KPS score, patients with a higher KPS score demonstrated better survival data. This was clinically significant in the BVZ cohort; however, it does not signify that these will be the only patients who will benefit from BVZ treatment.

Finally, analysis of the type of surgical resection demonstrated that the OS data was better in patients who underwent complete resection, with the exception of the BVZ cohort, where patients who were biopsied had the longest median OS. However, there were only two patients in this subgroup and the data should not be considered.

In this study, based on the results obtained, we can conclude that the BVZ/CPT-11 regimen is effective as a second-line treatment of primary GBM. According to our analysis, we can predict that patients with a higher KPS, lower age and complete 
resection may have a longer life expectancy; although, we should not select these patients alone as the sole beneficiaries of second-line treatment with BVZ-CPT11. Cost-effectiveness studies should be conducted to ascertain whether the cost of treatment is sustainable when there are budget constraints.

\section{References}

1. Louis DN, Ohgaki H, Wiestler OD, Cavenee WK, et al: The 2007 WHO classification of tumours of the central nervous system. Acta Neuropathol 114: 97-109, 2007.

2. Gurney JG and Kadan-Lottick N: Brain and other central nervous system tumors: rates, trends, and epidemiology. Curr Opin Oncol 13: 160-166, 2010.

3. Brem SS, Bierman PJ, Brem H, et al: Central nervous system cancers. J Natl Compr Canc Netw 9: 352-400, 2011.

4. Laigle-Donadey F and Delattre JY: Glioblastoma in the elderly. Geriatr Psychol Neuropsychiatr Vieil 9: 101-106, 2011 (In French).

5. Baldi I, Huchet A, Bauchet L and Loiseau H: Epidemiology of glioblastoma. Neurochirurgie 56: 433-440, 2010.

6. Bauchet L, Mathieu-Daudé H, Fabbro-Peray P, et al; Société Française de Neurochirurgie (SFNC); Club de Neuro-Oncologie of the Société Française de Neurochirurgie (CNO-SFNC); Société Française de Neuropathologie (SFNP); Association des Neuro-Oncologues d'Expression Française (ANOCEF): Oncological patterns of care and outcome for 952 patients with newly diagnosed glioblastoma in 2004. Neuro Oncol 12: 725-735, 2010 .

7. Deorah S, Lynch CF, Sibenaller ZA and Ryken TC: Trends in brain cancer incidence and survival in the United States: surveillance, epidemiology, and end results program, 1973 to 2001. Neurosurg Focus 20: E1, 2010.

8. HUCA tumour register. 2008 Annual Report. Hospital Universitario Central de Asturias and Instituto Universitario Oncológico, Principality of Asturias, 2008. ISSN 1138-8501. http://10.15.65.11/huca/web/contenidos/servicios/rt/rt2008/rt2008. pdf. Accesed January 21, 2012.

9. Stupp R, Mason WP, van den Bent MJ, et al; European Organisation for Research and Treatment of Cancer Brain Tumor and Radiotherapy Groups; National Cancer Institute of Canada Clinical Trials Group: Radiotherapy plus concomitant and adjuvant temozolamide for glioblastoma. N Engl J Med 352: 987-996, 2005.

10. Stupp R, Hegi ME, Mason WP, et al; European Organisation for Research and Treatment of Cancer Brain Tumour and Radiation Oncology Groups; National Cancer Institute of Canada Clinical Trials Group: Effects of radiotherapy with concomitant and adjuvant temozolomide versus radiotherapy alone on survival in glioblastoma in a randomised phase III study: 5-year analysis of the EORTC-NCIC trial. Lancet Oncol 10: 459-466, 2009.

11. Lacroix M, Abi-Said D, Fourney DR, et al: A multivariate analysis of 416 patients with glioblastoma multiforme: prognosis, extent of resection, and survival. J Neurosurg 95: 190-198, 2001.

12. Mineo JF, Bordron A, Baroncini M, et al: Prognosis factors of survival time in patients with glioblastoma multiforme: a multivariate analysis of 340 patients. Acta Neurochir (Wien) 149: 245-253, 2007.

13. Brandes AA, Tosoni A, Franceschi E, Reni M, Gatta G and Vecht C: Glioblastoma in adults. Crit Rev Oncol Hematol 67: 139-152, 2008.

14. Maxwell M, Naber SP, Wolfe HJ, Hedley-Whyte ET, Galanopoulos T, Neville-Golden J and Antoniades HN: Expression of angiogenic growth factor genes in primary human astrocytomas may contribute to their growth and progression. Cancer Res 51: 1345-1351, 1991.

15. Takahasi JA, Fukumoto M, Igarashi K, Oda Y, Kikuchi H and Hatanaka M: Correlation of Basic fibroblast growth factor expression levels with the degree of malignancy and vascularity in human gliomas. J Neurosurg 76: 792-798, 1992.

16. Folkman J: Tumor angiogenesis: Therapeutic implications. N Engl J Med 285: 1182-1186, 1971.
17. Friedman HS, Prados MD, Wen PY, et al: Bevacizumab alone and in combination with irinotecan in recurrent glioblastoma. J Clin Oncol 27: 4733-4740, 2009.

18. Norden AD, Young GS, Setayesh K, et al: Bevacizumab for recurrent malignant gliomas, efficacy, toxicity and patterns of recurrence. Neurology 70: 779-787, 2008.

19. Kreisl TN, Kim L, Moore K, et al: Phase II trial of single-agent bevacizumab followed by bevacizumab plus irinotecan at tumor progression in recurrent glioblastoma. J Clin Oncol 27: 740-745, 2009.

20. Vredenburgh JJ, Desjardins A, Herndon JE, et al: Bevacizumab plus irinotecan in recurrent glioblastoma multiforme. J Clin Oncol 25: 4722-4729, 2007.

21. Zuniga RM, Torcuator R, Jain R, et al: Efficacy, safety and patterns of response and recurrence in patients with recurrent high-grade gliomas treated with bevacizumab plus irinotecan. J Neurooncol 91: 329-336, 2009.

22. Stark-Vance V: Bevacizumab and CPT-11 in the treatment of relapsed malignant glioma. Neuro Oncol 7 (Suppl): 369, abstr 342, 2005.

23. Raval S, Hwang S and Dorsett L: Bevacizumab and irinotecan in patients with recurrent glioblastoma multiforme. J Clin Oncol 25 (Suppl): abstr 2078, 2007.

24. Vredenburgh JJ, Desjardins A, Herndon JE II, et al: Phase II trial of bevacizumab and irinotecan in recurrent malignant glioma. Clin Cancer Res 13: 1253-1259, 2007.

25. Ali SA, McHayleh WM, Ahmad A, et al: Bevacizumab and irinotecan therapy in glioblastoma multiforme: a series of 13 cases. J Neurosurg 109: 268-272, 2008.

26. Bokstein F, Shpigel S and Blumenthal DT: Treatment with bevacizumab and irinotecan for recurrent high-grade glial tumors. Cancer 112: 2267-2273, 2008.

27. Cloughesy TF, Prados MD, Wen PY, et al: A phase II, randomized, non-comparative clinical trial of the effect of bevacizumab (BV) alone or in combination with irinotecan (CPT) on 6-month progression free survival (PFS6) in recurrent, treatment refractory glioblastoma (GBM). J Clin Oncol 26 (Suppl): abstr 2010b, 2008

28. Desjardins A, Reardon DA, Herndon JE II, et al: Bevacizumab plus irinotecan in recurrent WHO grade 3 malignant gliomas. Clin Cancer Res 14: 7068-7073, 2008.

29. Guiu S, Taillibert S, Chinot O, et al: Bevacizumab/irinotecan. An active treatment for recurrent high grade gliomas: preliminary results of an ANOCEF Multicenter Study. Rev Neurol (Paris) 164: 588-594, 2008 (In French).

30. Gilbert MR, Wang M, Aldape K, et al: RTOG 0625: A phase II study of bevacizumab with irinotecan in recurrent glioblastoma (GBM). J Clin Oncol 27 (Suppl): abstr 2011, 2009.

31. Nghiemphu PL, Liu W, Lee Y, et al: Bevacizumab and chemotherapy for recurrent glioblastoma: a single-institution experience. Neurology 72: 1217-1222, 2009.

32. Poulsen HS, Grunnet K, Sorensen M, et al: Bevacizumab plus irinotecan in the treatment patients with progressive recurrent malignant brain tumours. Acta Oncol 48: 52-58, 2009.

33. Cloughesy T, Vredenburgh JJ, Day B, et al: Updated safety and survival of patients with relapsed glioblastoma treated with bevacizumab in the BRAIN study. J Clin Oncol 28 (Suppl): abstr 2008, 2010.

34. Desjardins A, Vredenburgh JJ, Reardon DA, et al: Longterm survival from the initial trial of bevacizumab and irinotecan. J Clin Oncol 28 (Suppl): abstr 2045, 2010.

35. Cohen MH, Shen YL, Keegan P and Pazdur R: FDA drug approval summary: bevacizumab (Avastin) as treatment of recurrent glioblastoma multiforme. Oncologist 14: 1131-1138, 2009.

36. Seiz M, KraVt U, Freyschlag CF, et al: Long-term adjuvant administration of temozolomide in patients with glioblastoma multiforme: experience of a single institution. J Cancer Res Clin Oncol 136: 1691-1695, 2010. 